\title{
Adaptive optics imaging of QSO UM402 field
}

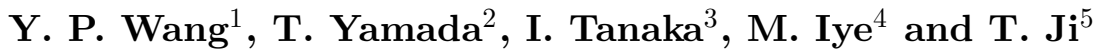 \\ ${ }^{1}$ Key Laboratory of Optical Astronomy, National Astronomical Observatories, CAS \\ ${ }^{2}$ Tohoku University, Japan ${ }^{3}$ Subaru telescope, National Astronomical Obs. of Japan \\ ${ }^{4}$ National Astronomical Obs. of Japan ${ }^{5}$ Polar Research Institute of China
}

\begin{abstract}
We present initial results from adaptive optics deep imaging in the Ks-band of QSO UM402 $\left(z_{e m}=2.856\right)$, with the IRCS camera on Subaru telescope.
\end{abstract}

Keywords. quasars: general

1) A faint companion galaxy $\left(m_{k}=21.91 \pm 0.26\right.$ in the Vega system) that lies $\sim 2$ ". 4 north of the QSO sightline has been clearly resolved by this high resolution imaging. The non-detection of this close companion by the previous deep R-band observation indicates that it has a red color $(R-K)_{V \text { ega }}>3.3$ (Guillenmin\& Bergeron 1997). This object has irregular morphology with two close components (separation $\sim 0$ "'.3). Given the small impact parameter $\left(b=19.6 \mathrm{kpc}\right.$, at $\left.z_{l l s}=2.531\right)$, it might be a candidate galaxy giving rise to the Lyman Limit system absorption at $z_{a b s}=2.531$ seen in the QSO spectrum.

2) Careful subtraction of the PSF from the QSO image revealed the QSO host galaxy. We model in detail the host galaxy properties using the 2-D decomposition algorithm GALFIT, and find that QSO UM402 is hosted by a giant elliptical of $m_{k}=20.19 \pm 0.15$ and of a scalelength $\sim 4 \mathrm{Kpc}$, as bright as other resolved hosts of radio-loud QSOs (RLQ) at similar redshift, although UM402 itself is radio quiet(RQQ) (Falomo et al. 2008).

3) We estimated the black hole mass of this QSO using the CIV broad emission line from SDSS spectra, adopting the prescription given by Vestergaard \& Peterson (2006). We found that UM402 is in good agreement with the $M_{B H}-\log L_{V}$ linear relation of the low-z bulges and ellipticals given by Dunlop et al. (2003), and other high-z QSO samples.

\section{Acknowledgements}

This project/publication was made possible through the support of a grant from the John Templeton Foundation and National Astronomical Observatories of Chinese Academy of Sciences. The opinions expressed in this publication are those of the author(s) do not necessarily reflect the views of the John Templeton Foundation or National Astronomical Observatories of Chinese Academy of Sciences. The funds from John Templeton Foundation were awarded in a grant to The University of Chicago which also managed the program in conjunction with National Astronomical Observatories, Chinese Academy of Sciences.

\section{References}

Dunlop, J. S., McLure, R. J. et al., 2003, MNRAS 340, 1095

Falomo, R., Treves, A., Kotilainen, J. K. et al., 2008, ApJ 673, 694

Guillenmin, P. \& Bergeron, J., 1997, A\&A 328, 499

Vestergaard, M. \& Peterson, B. M. 2006, ApJ, 641, 689 